\title{
La educación médica continua como herramienta constante de aprendizaje*
}

\author{
Dr. NICOLÁS VELASCO M. ${ }^{1}$ \\ 1 Decano Facultad de Medicina, Universidad Católica del Norte, Coquimbo. \\ Chile.
}

\begin{abstract}
Continuing medical education (CME) as a permanent tool for learning

CME is a challenge that our Universities have to assume not only after they give the final degree, although this must be done since students' begin their studies, due the changing scenarios that they are exposed. The increasing number of students that have now access to university studies, with important deficiencies in their formal education, but with high expectative, challenge universities to innovate in curriculums based competences, using expensive technological resources in order to give answers to the increasing demands, according to the expectative given by Chile in their international commitments. Is in this context that universities becomes the principal provider of qualified human resources that is demanded by our society for his development in a globalised world, where professionals, not only in health area, must be able to use this competences for a satisfactory performance. For a high quality CME, is of relevance that universities with their Medical Education Offices, must work together with Scientific Societies and with the different institution that works in health. For this purpose is of great importance that these institutions must know, not only the new scenarios where workers are involved, but they are in front of professionals with highly self instructions aptitudes, low time to spent in education but with multiple sources of information that challenge educators to be highly qualified, with newly concepts to assess their educational process and be in a permanent improvement of their own necessities to improve their own competences.
\end{abstract}

Key words: Continuing medical education, universities, graduate.

\section{Resumen}

La Educación Médica Continua (EMC) es un desafío que deben asumir las universidades no sólo después de recibido el título universitario, sino desde el momento mismo que ingresan los estudiantes a sus aulas dado los escenarios cambiantes a los que ésta se ve enfrentada. El aumento explosivo del número de estudiantes que ingresan en la actualidad, con importantes carencias de formación pero con un alto grado de exigencias; las obliga a innovar no sólo en modificar sus currículos en base a competencias, incorporando costosos recursos informáticos; sino que responder a las altas exigencias a las que, además, se ven enfren-

*Recibido el 17 de octubre de 2012 y aceptado para publicación el 6 de noviembre de 2012.

Los autores no refieren conflictos de interés.

Correspondencia: Dr. Nicolás Velasco M. Calle Larrondo 1281 Coquimbo, Chile. nvelasco@ucn.cl 
tadas para desenvolverse en un nivel equivalente al de los países con los cuales Chile se ha comprometido internacionalmente. En este contexto, ellas pasan a ser uno de los principales proveedores de un recurso humano calificado que exige la sociedad para desarrollarse en un mundo cada vez más globalizado, donde los profesionales que egresan, no sólo en el área de la salud, deben salir con nuevas competencias para enfrentar el entorno. En el área de la medicina, para una Educación Médica continua de calidad, pasa a tener gran relevancia el trabajo mancomunado que deben efectuar las Universidades a través de sus Oficinas de Educación Médica junto con las Sociedades Científicas y los diversos entes que participan en salud; lo que implica conocer a fondo no sólo los nuevos escenarios a los que se ven enfrentadas, sino actuar frente a profesionales con altas capacidades de auto instrucción, que manejan tiempos limitados de información y con acceso a múltiples fuentes de información masivas. De ahí el desafío de tener educadores altamente calificados, que evalúen adecuadamente los resultados de los procesos de formación y que estén en permanente búsqueda de sus propias necesidades de aprendizaje para mejorar sus competencias y por ende las de sus educandos.

Palabras clave: Educación médica continua, universidades, postgrado, postítulo.

Sin duda, la educación médica continua (EMC) es un imperativo en nuestros tiempos. Esta se podría definir como todo medio, o conjunto de ellos, dirigidos a cambiar la conducta de un médico de manera de mejorar permanentemente los resultados de su actuar con los pacientes, individualmente, en una comunidad o en un sistema de salud ${ }^{1}$.

No obstante es importante enfatizar que dicha Educación Médica no es algo que comience una vez recibido el título de Médico Cirujano, sino que es un tema que debe comenzar desde el ingreso mismo de los estudiantes de enseñanza media a la Universidad.

En ese sentido las Universidades son las primeras que deben tomar este desafío ya que se ven enfrentadas a un nuevo escenario para la educación que pueden resumirse en cuatro puntos principales:

\section{El rol que las universidades deben desempeñar en el mundo actual}

Las Universidades han debido pasar desde un trabajo centrado principalmente en el conocimiento puro, el que nunca dejará de existir, al de un conocimiento aplicado, en donde constantemente deben competir por la obtención de fondos públicos para su desarrollo. Estos se adjudican, en general, a proyectos que incluyen el concepto de $\mathrm{I}+\mathrm{D}+\mathrm{i}$ donde están incluidos tanto el desarrollo como la innovación. Este es un requisito que compete en general en la actualidad, a gran parte de los proyecto de investigación incluidos aquellos concernientes al área médica (Fondef y cols) ${ }^{2}$.

Por otro lado, toda Universidad está inserta dentro de un país el cual tiene un determinado contexto de desarrollo económico y social, de ahí que ellas deben también responder al modelo económico donde está inmersa lo que las obliga, en cierto modo, a una integración entre ella y dicho modelo, aunque este puede que no sea siempre de su agrado.
De igual forma, las Universidades son una importante fuente de recurso humano calificado. La formación de capital humano avanzado, así como la investigación, el desarrollo y la innovación constituyen en la actualidad sus pilares fundamentales ${ }^{3}$.

Las instituciones de educación superior deben entonces constituirse en una fuente esencial de oportunidades de formación continua y movilidad social. Si hace un par de décadas en nuestro país, entraban 20.000 ó 30.000 estudiantes a la educación superior al primer año; actualmente cada año la educación superior ofrece sobre 377.000 vacantes, siendo la oferta de las universidades cercana a las 160.000 vacantes, correspondiendo la diferencia a Institutos profesionales (IP) y Centros de Formación Técnica. Los matriculados, por otra parte, en Universidades el año 2011 alcanzaron a 156.555 estudiantes. El número total de estudiantes en Educación Superior en el año 2011, llegó a 978.000 alumnos de los cuales 597.000 correspondieron a Universidades $(61 \%)$ y de éstas el $33 \%$ a Universidades privadas y $28 \%$ a universidades del CRUCH. Esta proyección seguirá aumentando en los próximos años ${ }^{4}$.

Lo anterior ha llevado a que la educación superior se comience a comportar como una verdadera industria en la producción de profesionales donde la medicina no está exenta. Las carreras de medicina que en la década del 70 eran impartidas por 6 universidades; en la actualidad nos encontramos que existen 30 carreras de medicina impartidas por 23 universidades en distintos lugares del país, de acuerdo a datos de ASOFAMECH y Consejo Nacional de educación ${ }^{5}$.

Pero, las instituciones universitarias deben velar por mejorar constantemente la calidad y pertinencia de su oferta académica; cuestión de la mayor importancia para generar programas de formación e investigación que efectivamente impacten sobre la capacidad competitiva del país y permitan la inserción exitosa también de jóvenes de familias 
vulnerables al mercado del trabajo, muchos de los cuales son primera generación universitaria familiar.

\section{Los cambios culturales que influyen en la educación superior y en la EMC}

En este aspecto vuelve a tomar relevancia el impacto del número de estudiantes que ingresan a la educación superior ya que al crecer exponencialmente el número de éstos; nos enfrentamos también a estudiantes con una diversidad muy diferente a la de décadas anteriores, donde existen carencias importantes tanto educacionales como socio económicas y afectivas que sin duda influyen tanto en las tasas de retención como de deserción, punto al cual debemos mirar con preocupación. Igualmente, el nivel de exigencia, de parte de los estudiantes, hacia la Universidad por el servicio que están adquiriendo es cada vez mayor.

Por otro lado, tenemos las demandas del "consumidor" llámese industria, comercio, municipios etc., las que son cada día crecientes en cuanto al tipo de profesional que requiere, lo que nos obliga a perfeccionar los sistemas de educación ya que se requieren profesionales que desde su egreso dominen una serie de variables que antes se adquirían con el correr de los años.

Los sistemas productivos y también los sanitarios, desde el punto de vista tecnológico, están también en constante evolución. No en vano cuando debemos formular los perfiles de egreso de nuestros estudiantes; se hace imprescindible en la actualidad, consultar tanto a la comunidad como a los empleadores para poder entregar el profesional que la sociedad demanda y no el profesional que nosotros creemos que la comunidad necesita. De ahí el imperativo de que formemos el recurso humano acorde a esta sociedad que cambia rápidamente.

El gran desarrollo de las comunicaciones nos obliga a incorporar las nuevas tecnologías de enseñanza, lo que encarece por un lado este proceso, y por otro pasan a ser de rápida obsolescencia.

\section{Los cambios en el rol de los consumidores- estudiantes y/o empleadores}

Los estudiantes se dirigirán, probablemente, a aquella Universidad que les ofrezca el mejor resultado acorde a los medios por él disponibles y dependiendo del número de alternativas que ofrezcan las distintas casas de estudio. De ahí que la exigencia de las acreditaciones pasan a jugar un rol relevante en nuestra área médica y la importancia de que estas sean transparentes y realmente permitan al "consumidor" tener la certeza de que lo que se ofrece, es realmente lo que él quiere comprar. La aparición de superintendencias de distinta índole que deben regular este tema tan sensible, creemos es un avance importante en pos de la calidad.

Por otro lado, el rol de los medios de comunicación ha pasado a tener una influencia marcada en cuanto a la fiscalización de lo que se ofrece como de la oportunidad de la oferta, así como también para informar al público de las diversas alternativas existentes no sólo a nivel regional o nacional sino también con los cambios externos. El estar nuestro país en la OCDE, implica mejorar en calidad y oportunidad de los servicios que se ofrecen incluyendo a los educadores. Aparecen rankings comparativos tanto dentro del ámbito nacional como con el extranjero, a los cuales las facultades de medicina y los medios de comunicación no están ajenos.

\section{Los cambios en la metodología de la enseñanza}

Tradicionalmente la formación del médico estaba centrada en un período inicial de formación en ciencias básicas con un contacto relativamente tardío con la clínica y donde las principales habilidades profesionales se enseñaban en la segunda mitad de la formación médica; este entrenamiento se daba preferentemente en hospitales de alta complejidad lo que llevaba a una precoz especialización y una limitada experiencia tanto en atención primaria o comunitaria así como del trabajo en equipo.

Los cambios señalados anteriormente han obligado a las universidades a renovar sus diseños curriculares poniendo énfasis en currículos por competencias, definiendo claramente cuáles son estas competencias, con integración tanto vertical como horizontal; que tuviesen un contacto más temprano con la clínica así como también una mayor orientación comunitaria. Esto necesariamente lleva a cambiar los métodos pedagógicos, aplicar nuevas tecnologías que resulten más atractivas para los estudiantes, donde el foco del proceso enseñanza aprendizaje pasa del profesor al estudiante. Los docentes deben realmente adquirir habilidades pedagógicas que antes eran netamente intuitivas, llevándolos a perfeccionarse tanto en técnicas de enseñanza más personalizadas y atractivas, como principalmente en sistemas evaluativos objetivos y estandarizados que demuestran ser más equitativos, menos subjetivos ${ }^{5}$ y donde la enseñanza además entregue un componente de gestión fuerte, acorde a las exigencias del medio actual.

Todo lo anterior, ha llevado a que nuestra base para una educación médica continua en el pos grado, deba enfocarse hacia profesionales que son cada vez más autodidactas, capaces de buscar por si mismo información relevante tanto para resolver los pro- 
blemas ya sea clínicos como de la comunidad ${ }^{6}$, con pensamiento crítico, capaces de trabajar en equipo y con sentido comunitario, en donde las habilidades de comunicación deben jugar un rol preponderante así como también las habilidades o competencias en gestión, las que deben estar acordes con un manejo racional de los recursos.

Con todos estos antecedentes, y probablemente muchos otros, que pueden ser largo de enumerar en este documento, llegamos al egresado de medicina que se insertará en el medio ya sea hospitalario o comunitario.

\section{En qué consiste el desarrollo profesional del médico}

De acuerdo a la Academia de Medicina de USA; éste es un continuo a lo largo de su vida profesional que se construye mediante oportunidades formales como informales de allegar conocimiento médico nuevo; en que aplica innovación en su práctica diaria mejorando su accionar? ${ }^{7}$.

De ahí que la EMC debe estar acorde a los cambios sanitarios como también a los distintos medios de aprendizaje existentes. Además debe estar orientada a satisfacer las necesidades de los profesionales lo que implica consultar a los mismos profesionales sobre sus necesidades de aprendizaje de modo que ellos desarrollen su propia responsabilidad de aprender con el fin de mejorar continuamente su desempeño ${ }^{8}$.

La educación de sólo pregrado no es un certificado de competencia vitalicia.

La EMC debe ser considerada como la etapa final y más larga en la formación de un médico ${ }^{7}$, para ello, insistimos, debe iniciarse en el pregrado de la escuela de Medicina, perfeccionarse en el postítulo y seguir durante toda la vida activa del médico ${ }^{1}$.

Ningún médico puede pretender permanecer vigente sin un programa de aprendizaje activo, continuo y efectivo, y no cabe la menor duda que esta exigencia y la velocidad de obsolescencia tanto de los conocimientos médicos así como de las técnicas aplicadas aumentarán progresivamente esta necesidad de aprendizaje $e^{9,10}$.

Los nuevos conocimientos, la introducción de nuevas tecnologías, los cambios de los perfiles epidemiológicos, así como la aparición de enfermedades crónicas junto al envejecimiento de la población y la gran cantidad de información disponible tanto en revistas científicas como a través de los medios de comunicación, donde nuestros pacientes también están cada vez más informados, obliga a los profesionales a estar en constante revisión de sus procedimientos.

Por otro lado, las distintas organizaciones de profesionales, sociedades científicas, universidades y servicios de salud deben trabajar en forma mancomunada en pos de entregar a los profesionales una educación efectiva y de calidad con el fin de que éstos mejoren su accionar frente a la comunidad y a los pacientes?

Dentro de esta educación efectiva, es importante tener presente que debe buscarse lo que sea más adecuado para el educando; esto significa que ésta debe ser buscando su mayor conveniencia, lo que implica que debe ser en lo posible en el lugar adecuado, en el tiempo adecuado y a un ritmo de aprendizaje determinado ya que no todos los profesionales tienen, a veces, ni el tiempo suficiente disponible o las facilidades de movilización a un centro de EMC respecto a su lugar de trabajo y sobre todo no todos tienen similares velocidades de aprendizaje.

La relevancia de lo que se enseña pasa a ser primordial y en ella deben tratar de conjugarse los tres componentes que tiene la relevancia; lo que se enseña, o sea los contenidos del programa, las competencias que se quieren entregar y las deficiencias que tiene el profesional y que éste busca mejorar ${ }^{11}$. En términos prácticos es importante hacerse la pregunta de cómo, cuándo y por qué aprenden los médicos.

Probablemente para respondernos estas preguntas no vamos a encontrar una sola respuesta. Cada profesional tiene métodos diferentes de aprendizaje y estos también son variables acorde a las circunstancias en que éste se está desenvolviendo; de ahí que en ocasiones podrá ser en base a resolución de problemas específicos, o casos clínicos concretos, o bien en forma informal en conversaciones con otros profesionales; por otro lado, pueden existir motivaciones particulares como de reconocimiento profesional, o porque se busca una recertificación o incluso por estímulos económicos que acompañan a la práctica médica. De ahí que en general, los médicos progresan a su propio ritmo dependiendo de la motivación, su conocimiento o percepción del problema o de las carencias específicas que tiene así como de las oportunidades de perfeccionamiento y de la necesidad de poder aplicar lo aprendido ${ }^{12}$. De ahí la importancia de la permanente consulta a los profesionales de averiguar cuáles son sus verdaderas necesidades de aprendizaje ${ }^{13}$.

El $e$ - learning ofrece en la actualidad a los profesionales un fácil control tanto sobre los contenidos como sobre la secuencia y rapidez de aprendizaje acorde a las capacidades personales y el tiempo disponible, permitiendo además diseñar los propios objetivos de aprendizaje ${ }^{14}$.

Es así como en la actualidad internet con la gran cantidad de información de que dispone y la rapidez de comunicación y su fácil acceso; ha pasado a ser una de las fuentes primordiales de EMC de los médicos. 
A este respecto, la principal razón de consulta se debe a la búsqueda de solución para un paciente o caso particular determinado de la vida diaria. La credibilidad de la fuente de información así como la facilidad y rapidez de acceso a la fuente a través de internet es lo más relevante para el médico en la actualidad $^{15}$. Aquellas barreras que se aprecian en este caso incluyen, a veces, el exceso de información irrelevante y poco específica para responder a una pregunta específica.

Todo lo anteriormente mencionado debe tomarse en cuenta entre los diferentes entes formadores en pos grado y EMC. La articulación de las diferentes organizaciones de educación médica se hace necesaria para obtener los mejores resultados y tratar de abarcar las diferentes condiciones y lugares donde se desenvuelven los profesionales.

Desgraciadamente, la investigación en educación médica ha sido, en general, de bajo impacto ya que los investigadores tienden a abordar temas en forma independiente acorde a sus propios intereses particulares y no a programas predefinidos. Se deben buscar líneas de investigación que sean realmente un aporte al progreso de los médicos en cuanto a sus competencias.

Ningún proceso de EMC puede ser satisfactorio si no va acompañado de una evaluación de resultados de la enseñanza impartida o de los cambios de conducta en los profesionales pues lo que importa finalmente es el cambio en las conductas y que estos cambios se traduzcan en mejores resultados a nivel de los pacientes o de los sistemas ${ }^{16}$. Los indicadores usados para medir si la estrategia adoptada y las herramientas elegidas producen o no el cambio deseado, son cruciales en cualquier sistema de educación médica ${ }^{17}$.

Esto obliga a los educadores médicos y a las oficinas de educación médica, a buscar sus propias necesidades de aprendizaje para mejorar sus competencias, que permitan prolongar sus capacidades de enseñanza y desarrollar las estrategias que muestren novedades que motiven al aprendizaje. Paralelamente a esto, deben investigar como aprenden los médicos para así promover los cambios en sus comportamientos. Promover actividades profesionales para los médicos que sean acordes o útiles para su desempeño profesional.

Por otro lado, se deben proveer los recursos necesarios y adecuados para que ellos puedan ampliar sus destrezas de aprendizaje proveyendo las actividades y servicios de la más alta calidad y se debe buscar desarrollar nuevas metodologías para medir estos logros de aprendizaje 7 .

Finalmente, debe promoverse el desarrollo de los educadores en salud, que incluya el reconocimiento tanto de la teoría como de la investigación, que sus- tenten los cambios apropiados en el conocimiento médico para el desarrollo de nuevas competencias que permita mejoras sustanciales en los distintos ámbitos de la salud.

\section{Referencias}

1. Dagnino J. Educación Médica Continua. Ars Médica 2007; $15: 15$.

2. http://www.fondef.cl/content/view/14/103/(concursos estatales).

3. Rodríguez-Ponce E . El rol de las Universidades en la Sociedad del conocimiento y en la era de la Globalización: Evidencias en Chile. Interciencia 2009;34:822829. versión impresa ISSN 0378-1844

4. Sistema de educación superior 2007-2011. Dirección de análisis institucional Universidad de la Frontera.

5. Estadísticas y bases de datos, carreras y programas Consejo nacional de educación: www.cned.cl

6. Manning PR, De Bakey L. Continuing Medical Education: The Paradigm Is Changing. J Continuing Education Health Prof. 2001;21:46-54.

7. Bennett NL, Davis DA, Easterling W Friedmann P, Green JS, Koeppen BM, et al. Continuing Medical Education: A New Vision of the Professional Development of Physicians. Academic Medicine 2000;75:1167-72.

8. Slotnick HB. Physician's Learning Strategies. Chest 2000;118:18-23.

9. Norman GR, Shannon SI, Marrin ML. The need for needs assessment in continuing medical education. BMJ 2004;328:999-1001.

10. Donen N. No to mandatory continuing medical education, Yes to mandatory practice auditing and professional educational development. CMAJ 1998;158:1044-6.

11. Harden RM, Laidlaw JM. Effective Continuing Education: The Crisis Criteria. AMEE Education Guide 2006;4:3-16.

12. Mazmanian P, David AD. Continuing Medical Education and the Physician as a Learner: Guide to the Evidence. JAMA 2002;288:1057-60.

13. Grant J. Learning needs assessment: assessing the need. BMJ 2002;324:156-9.

14. Ruiz JG, Mintzer MJ, Leipzig RM. The Impact of ELearning in Medical Education. Academic Medicine 2006; 81:207-12.

15. Casebeer L, Bennett N, Kristofco R, Carillo A, Centor R. Physician internet medical information seeking and on-line continuing education use patterns. J Contin Educ Health Prof. 2002; 22:33-42.

16. Tian J, Atkinson NL, Portnoy B, Gold RS. A Systematic Review of Evaluation in Formal Continuing Medical Education. J Cont Educ Health Prof. 2007;27:16-27.

17. Harden RM, Grant J, Buckley G, Hart IR. BEME Guide $\mathrm{N}^{\mathrm{o}} 1$ : Best Evidence in Medical Education. Med Teacher. 1999;21:553-62. 